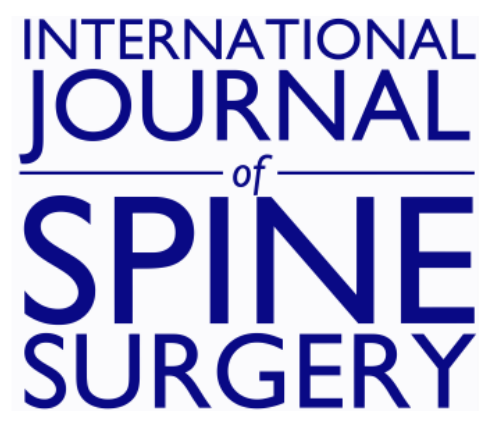

\title{
The Use of Patient-Reported Outcomes Measurement Information System in Spine: A Systematic Review
}

Kelsey Young, Michael Steinhaus, Catherine Gang, Avani Vaishnav, Bridget Jivanelli, Francis Lovecchio, Sheeraz Qureshi, Steven McAnany, Han Jo Kim and Sravisht Iyer

Int J Spine Surg 2021, 15 (1) 186-194

doi: https://doi.org/10.14444/8024

http://ijssurgery.com/content/15/1/186

This information is current as of April 26, 2023.

Email Alerts Receive free email-alerts when new articles cite this article. Sign up at:

http://ijssurgery.com/alerts

The International Journal of Spine Surgery

2397 Waterbury Circle, Suite 1,

Aurora, IL 60504, Phone: +1-630-375-1432 


\title{
The Use of Patient-Reported Outcomes Measurement Information System in Spine: A Systematic Review
}

\author{
KELSEY YOUNG, MD, ${ }^{1}$ MICHAEL STEINHAUS, $\mathrm{MD},{ }^{2}$ CATHERINE GANG, $\mathrm{MPH},{ }^{2}$ AVANI VAISHNAV, \\ MBBS, ${ }^{2}$ BRIDGET JIVANELLI, MILS, ${ }^{2}$ FRANCIS LOVECCHIO, MD,${ }^{2}$ SHEERAZ QURESHI, MD,${ }^{2}$ STEVEN \\ MCANANY, MD, ${ }^{2}$ HAN JO KIM, MD ${ }^{2}$ SRAVISHT IYER, MD ${ }^{2}$ \\ ${ }^{1}$ Department of Orthopaedic Surgery, University of Pennsylvania, Philadelphia, Pennsylvania, ${ }^{2}$ Hospital for Special Surgery, New York, New York
}

\begin{abstract}
Background: The Patient-Reported Outcomes Measurement Information System (PROMIS) was developed to provide an easily administered patient-outcome questionnaire that was adaptable to a variety of medical and surgical subspecialties. Numerous authors have examined the effectiveness of PROMIS in various areas of spine surgery. Our goal was to systematically review PROMIS scores compared with legacy patient-reported outcomes measures (PROMs) in spinal surgery and spine pathology.

Methods: A systematic search of the PubMed, EMBASE, and Cochrane databases using the Preferred Reporting Items for Systematic Reviews and Meta-Analysis (PRISMA) guidelines was performed, yielding 254 unique studies reporting on "PROMIS" in "spine." Each study was independently reviewed. A total of 16 studies were selected for inclusion.

Results: The pooled sample size yielded a total of 4268 patients. In the cervical population, PROMIS physical function (PF; $|\mathrm{r}|=.47-.87$, pain intensity (PIn; $|\mathrm{r}|=.61-.74$ ), pain interference (PIf; $|\mathrm{r}|=.65-.88$ ), and pain behavior (PB; $|\mathrm{r}|=.59-.74)$ correlated with the Neck Disability Index (NDI). PROMIS PF also strongly correlated with the modified Japanese Orthopaedic Association scale (mJOA; $|\mathrm{r}|=.61-.72$ ). Among patients with lumbar pathology and adult spinal deformities, PROMIS PF $(|\mathrm{r}|=.53-.85)$, PIn $(|\mathrm{r}|=.73-.78)$, PIf $(|\mathrm{r}|=.59-.89)$, and PB $(|\mathrm{r}|=.58-.82)$ strongly correlated with the Oswestry Disability Index (ODI). PF $(|\mathrm{r}|=.51-.78)$, PIf $(|\mathrm{r}|=.60-.70)$, and anxiety $(|\mathrm{r}|=.73)$ also strongly correlated with the Scoliosis Research Society (SRS)-22 and SRS-30. When comparing measures of global health, PROMIS PF was strongly correlated with the Short Form (SF)-12 and SF-36 $(|\mathrm{r}|=.50-.85)$. On average, all PROMIS domains required less time to complete (49.6-56 seconds) than the ODI (176 seconds), NDI (190.3 seconds), SF-12 (214 seconds), and SF-36 physical function domains ( 99 seconds). The responsiveness of the PROMIS PF, PIf, and PB was comparable to that of legacy measures ODI, NDI, and SF-12.
\end{abstract}

Conclusions: The PROMIS PF, PIn, PIf, and PB demonstrated moderate to strong correlations with NDI, mJOA, ODI, SRS, and SF-12 measures in various populations of spine patients. All PROMIS domains had decreased time to completion and similar responsiveness compared with legacy measures.

Level of Evidence: 2.

Clinical Relevance: These results highlight the potential of PROMIS as a valid and reliable tool to assess patientreported outcomes in spinal surgery patients and support more widespread use of PROMIS in spine.

Other \& Special Categories

Keywords: patient-reported outcome measures (PROMs), PROMIS, spine

\section{INTRODUCTION}

As the focus on evidence-based medicine and value-driven health care has grown over the past several decades, patient-reported outcomes measures (PROMs) have gained in popularity and importance in orthopedics. ${ }^{1,2}$ The current approach to PROMs focuses on a group of "legacy outcome measures" that measure outcomes in specific disease states and/or anatomical locations. ${ }^{3,4}$ This diseasespecific approach, however, is fragmented by its nature and makes it difficult to compare patient health and response with treatment across different pathologies and interventions. Furthermore, current PROMs are burdensome and require significant time to complete. ${ }^{5}$

To remedy these challenges, in 2004 the National Institute of Health developed the Patient-Reported Outcomes Measurement Information System (PROMIS) to provide a widely reliable and valid tool to measure patient outcomes across medicine. ${ }^{4}$ PROMIS questionnaires offer a set short-form 


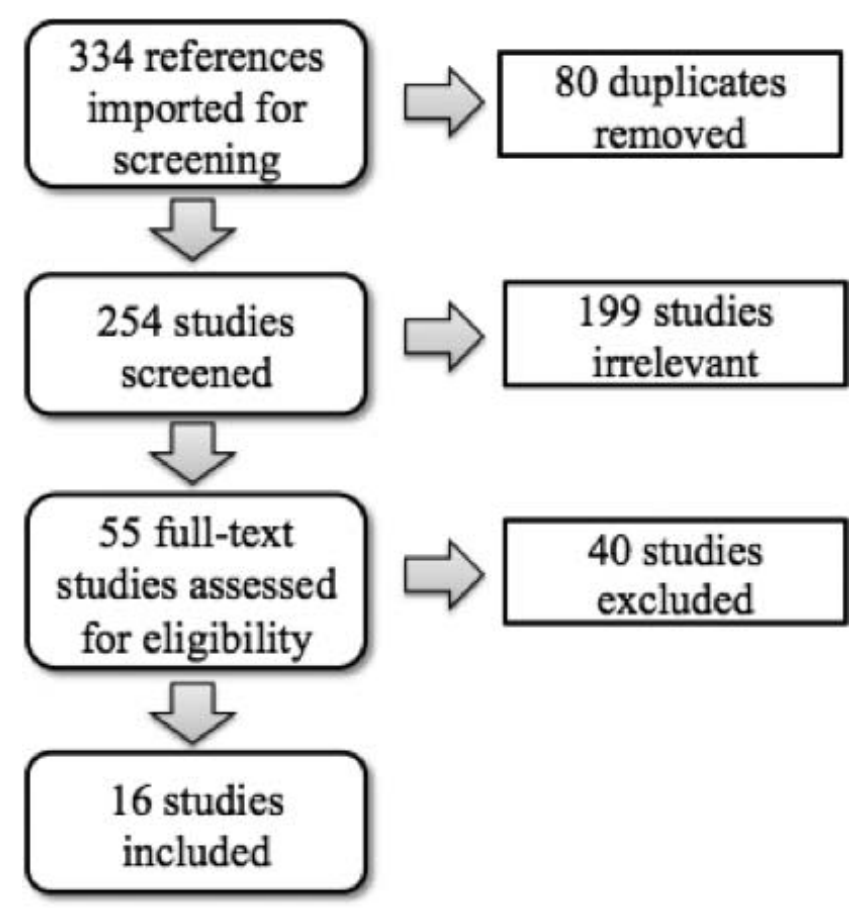

Figure. Flow diagram representing search process used in the study. A total of 334 references were identified from the initial search terms. Ultimately, 55 references underwent full text review and 16 studies were selected for final inclusion.

version with a fixed set of 4-10 questions or a computer adaptive test version of 4-12 questions tailored to individuals and the severity of their symptoms. ${ }^{6}$ Since its development, there has been a substantial increase in attention surrounding the validity, responsiveness, and ease of using PROMIS in spine patients.

The goal of this study was to systematically review PROMIS scores compared with legacy PROMs in spinal surgery. We hypothesize that PROMIS scores will be highly correlated with the various legacy PROMs in spinal surgery and be less burdensome to patients, requiring less time to complete.

\section{METHODS}

A systematic search of 3 databases, MEDLINE via PubMed, EMBASE, and the Cochrane Library, was performed to identify all relevant literature published between database inception and November 2018. The search strategy was created in collaboration with a professional medical librarian. Search terms included a combination of $\mathrm{MeSH}$ terms and keywords related to PROMIS and spine. The search was completed on November 20, 2018.
The complete search strategy is available in the Appendix.

Inclusion criteria were any orthopedic-related article with level of evidence 1-4 and reporting use of PROMIS in spine pathology. Articles not related to spine, non-English articles, unpublished studies, studies with level 5 evidence, letters to the editor, editorials, basic science articles, and conference abstracts were excluded. Covidence (Melbourne, Australia) software was used for the screening process. The search yielded 254 unique studies reporting on "PROMIS" in "spine." Each study was independently reviewed. Ultimately, 55 studies were selected for full-text review, and 16 studies were selected for final inclusion (see Figure). Study characteristics (eg, study population, level of evidence, number of included patients, mean age) were recorded. The reported outcome instruments were noted and correlations between PROMIS domains and legacy measures were documented for each study. Correlations were reported as weak $(0 \leq|\mathrm{r}|<$ $3)$, moderate $(3 \leq|\mathrm{r}| \leq 5)$, or strong $(|\mathrm{r}| \geq 5)$. Responsiveness of the instrument and time to completion were also noted when available.

\section{RESULTS}

The characteristics of the included studies are shown in Table 1. The pooled sample size from the 16 studies yielded a total of 4268 patients. Of the 16 studies, one was level 1; nine were level 2; four were level 3; and two were level 4. The mean number of patients in each study was 267 (range, 41-1607).

\section{Cervical Spine and Neck Pain}

Of the 16 studies included, 7 reported on cervical pathology and the respective legacy measures (Table 2). In this population, PROMIS physical function (PF; $|\mathrm{r}|=.47-.87$, pain intensity (PIn; $|\mathrm{r}|=.61-.74)$, pain interference (PIf; $|\mathrm{r}|=.65-.88$ ), and pain behavior (PB; $|\mathrm{r}|=.59-.74)$ all moderately to strongly correlated with the Neck Disability Index (NDI). In Table 2, correlations may be reported as negative, given that higher PROMIS PF scores indicating an improvement in physical functioning would correlate with lower disability scores such as the NDI. Owen et $\mathrm{al}^{16}$ also showed PROMIS physical function strongly correlated with the mJOA $(|\mathrm{r}|=.61-.72)$. In a population of nonsurgical patients, Moses et $\mathrm{al}^{15}$ demonstrated a strong correlation between PROMIS PF and the NDI ( $|\mathrm{r}|$ 
Table 1. Characteristics of the studies included in this systematic review.

\begin{tabular}{|c|c|c|c|c|c|c|}
\hline Study & Population & Level of Evidence & $\mathbf{n}$ & Mean age, $\mathbf{y}$ & PROMIS & Legacy Measure \\
\hline Bernstein et $\mathrm{al}^{7}$ & Adult spinal deformity & 3 & 163 & 48 & $\begin{array}{l}\text { Physical function } \\
\text { Pain interference } \\
\text { Depression }\end{array}$ & SRS-30 \\
\hline Bhatt et $\mathrm{al}^{8}$ & Lumbar discectomy & 2 & 78 & 41.6 & $\begin{array}{c}\text { Physical function } \\
\text { Pain intensity } \\
\text { Pain behavior }\end{array}$ & $\begin{array}{l}\text { ODI } \\
\text { SF-12 }\end{array}$ \\
\hline Boody et $\mathrm{al}^{9}$ & Cervical spine surgery & 1 & 59 & 55.7 & $\begin{array}{c}\text { Physical function } \\
\text { Pain intensity } \\
\text { Pain behavior }\end{array}$ & $\begin{array}{l}\text { NDI } \\
\text { SF-12 }\end{array}$ \\
\hline Brodke et al $^{10}$ & Back/leg pain & 2 & 1607 & 54.2 & Physical function & $\begin{array}{l}\text { SF-36 PFD } \\
\text { ODI }\end{array}$ \\
\hline Haws et $\mathrm{al}^{11}$ & MIS TLIF & 2 & 74 & 53.9 & Physical function & ODI \\
\hline Hung et $\mathrm{al}^{12}$ & Spine clinic & 3 & 316 & 57 & $\begin{array}{l}\text { Anxiety } \\
\text { Depression }\end{array}$ & $\mathrm{mZDI}$ \\
\hline Hung et $\mathrm{al}^{13}$ & Spinal disorders & 2 & 763 & 58.28 & $\begin{array}{l}\text { Physical function } \\
\text { Pain interference }\end{array}$ & $\begin{array}{l}\text { ODI } \\
\text { NDI }\end{array}$ \\
\hline Khechen et al ${ }^{14}$ & MIS Lumbar Microdiscectomy & 4 & 41 & 50.3 & Physical function & $\begin{array}{l}\text { ODI } \\
\text { VAS Back } \\
\text { VAS Leg }\end{array}$ \\
\hline Moses et $\mathrm{al}^{15}$ & Neck pain & 3 & 130 & $45-55$ & $\begin{array}{c}\text { Physical function } \\
\text { Pain intensity } \\
\text { Pain interference }\end{array}$ & $\begin{array}{l}\text { NDI } \\
\text { VAS Back } \\
\text { VAS Neck } \\
\text { VAS Arm } \\
\text { VAS Leg }\end{array}$ \\
\hline Owen et $\mathrm{al}^{16}$ & Cervical myelopathy surgery & 3 & 60 & 60 & $\begin{array}{l}\text { Pain intensity } \\
\text { Anxiety } \\
\text { Depression }\end{array}$ & $\begin{array}{l}\text { NDI } \\
\text { mJOA } \\
\text { GAD-7 } \\
\text { PHQ-8 }\end{array}$ \\
\hline Papuga et al ${ }^{17}$ & Spine clinic & 4 & 283 & 55.2 & $\begin{array}{l}\text { Physical function } \\
\text { Pain interference } \\
\text { Pain behavior }\end{array}$ & $\begin{array}{l}\text { ODI } \\
\text { NDI }\end{array}$ \\
\hline Patel et al ${ }^{18}$ & Lumbar stenosis surgery & 2 & 98 & 61.9 & $\begin{array}{l}\text { Physical function } \\
\text { Pain interference } \\
\text { Pain behavior }\end{array}$ & $\begin{array}{l}\text { ODI } \\
\text { ZCQ } \\
\text { SF-12 }\end{array}$ \\
\hline Purvis et $\mathrm{al}^{19}$ & Anterior cervical spine surgery & 2 & 148 & 53 & $\begin{array}{l}\text { Physical function } \\
\text { Pain intensity } \\
\text { Anxiety } \\
\text { Depression }\end{array}$ & $\begin{array}{l}\text { NDI } \\
\text { GAD-7 } \\
\text { PHQ-8 }\end{array}$ \\
\hline Purvis et $\mathrm{al}^{20}$ & Lumbar disc degeneration decompression & 2 & 231 & 59 & $\begin{array}{l}\text { Physical function } \\
\text { Pain intensity } \\
\text { Anxiety } \\
\text { Depression }\end{array}$ & $\begin{array}{l}\text { ODI } \\
\text { SF-12 } \\
\text { BPI Pain } \\
\text { BPI Back } \\
\text { BPI Leg } \\
\text { GAD-7 } \\
\text { PHQ-8 }\end{array}$ \\
\hline Raad et $\mathrm{al}^{21}$ & Adult spinal deformity & 2 & 123 & 58 & $\begin{array}{l}\text { Physical function } \\
\text { Pain interference } \\
\text { Anxiety }\end{array}$ & $\begin{array}{l}\text { ODI } \\
\text { SRS-22r }\end{array}$ \\
\hline Sharma et $\mathrm{al}^{22}$ & Cervical/Lumbar surgery & 2 & 94 & 49 & Physical function & $\begin{array}{l}\text { ODI } \\
\text { NDI }\end{array}$ \\
\hline
\end{tabular}

Abbreviations: BPI, Brief Pain Inventory; GAD-7, General Anxiety Disorder-7; MIS, minimally invasive surgery; TLIF, transforaminal lumbar interbody fusion; mJOA, modified Japanese Orthopaedic Association scale; mZDI, modified Zung Depression Index; NDI, Neck DISABILITY INDEX; ODI, Oswestry Disability Index; PHQ-8, Patient Health Questionnaire; SF-12, Short Form 12; SF-36 PFD, Short Form 36 physical function domains; VAS, visual analog scale; SRS-22r, Scoliosis Research Society questionnaire; ZCQ, Zurich Claudication Questionnaire.

$=.771)$ but only moderate correlations with the visual analog scale (VAS) instruments $(|\mathrm{r}|=.302-$ $.428)$.

\section{Lumbar Spine and Adult Spinal Deformity}

There were 11 studies that reported on patients with lumbar pathology or adult spinal deformity. In this population, the most common legacy measures included the Oswestry Disability Index (ODI),
Scoliosis Research Society (SRS)-30 and SRS-22r, and VAS Back/Leg (Table 3). Among these patients, PROMIS PF $(|\mathrm{r}|=.53-.85)$, PIn $(|\mathrm{r}|=$ $.73-.78)$, PIf $(|\mathrm{r}|=.59-.89)$, and PB $(|\mathrm{r}|=.58-.82)$ all strongly correlated with the ODI. The PROMIS PF $(|\mathrm{r}|=.51-.78)$ and PIf $(|\mathrm{r}|=.60-.70)$ also strongly correlated with the SRS-22 and SRS-30, whereas PROMIS PF demonstrated a wide range of correlations with VAS Back $(|\mathrm{r}|=.09-.69)$ and with VAS Leg $(|\mathrm{r}|=.33-.64)$. 
Table 2. Correlations of various PROMIS domains with cervical spine legacy measures.

\begin{tabular}{|c|c|c|c|c|c|c|}
\hline Study & Population & n & PROMIS & Legacy & Correlation & Responsiveness \\
\hline \multicolumn{7}{|l|}{ Surgical Studies } \\
\hline \multirow[t]{3}{*}{ Boody et $\mathrm{al}^{9}$} & Cervical spine & 59 & PF & NDI & -.81 & \\
\hline & & & PIn & NDI & .61 & \\
\hline & & & PB & NDI & .59 & \\
\hline \multirow[t]{6}{*}{ Hung et $\mathrm{al}^{13}$} & Spinal disorders & 763 & $\mathrm{PF}$ & NDI Pre-op & -.66 & $\mathrm{PF} \mathrm{SRM}=1.31$ \\
\hline & & & & NDI $3 \mathrm{mo}$ & -.76 & PIf SRM $=1.16$ \\
\hline & & & & NDI 6 mo & -.80 & NDI SRM $=1.18$ \\
\hline & & & PIf & NDI Pre-op & .71 & \\
\hline & & & & NDI 3 mo & .81 & \\
\hline & & & & NDI 6 mo & .74 & \\
\hline \multirow{5}{*}{ Papuga et $\mathrm{al}^{17}$} & Spine clinic & $283^{\mathrm{a}}$ & PF & NDI Pre-op & .8334 & \\
\hline & & & & NDI Post-op & .871 & \\
\hline & & & PIf & NDI Pre-op & .6544 & \\
\hline & & & & NDI Post-op & .8875 & \\
\hline & & & PB & NDI Pre-op & .7367 & \\
\hline \multirow[t]{2}{*}{ Purvis et $\mathrm{al}^{19}$} & Anterior cervical spine & 148 & $\mathrm{PF}$ & NDI & -.47 & $\mathrm{PF} \mathrm{ES}=0.35$ \\
\hline & & & PIn & NDI & .74 & PIn ES $=0.86$ \\
\hline \multirow[t]{4}{*}{ Owen et $\mathrm{al}^{16}$} & Cervical myelopathy & 60 & $\mathrm{PF}$ & NDI Pre-op & -.69 & \\
\hline & & & & NDI 6 mo & -.76 & \\
\hline & & & & mJOA Pre-op & .61 & \\
\hline & & & & mJOA 6 mo & .72 & \\
\hline \multirow[t]{2}{*}{ Sharma et $\mathrm{al}^{22}$} & Cervical surgery & 42 & $\mathrm{PF}$ & NDI Pre-op & -.603 & $\mathrm{PF}$ SRM $=0.98$ \\
\hline & & & & NDI 3 mo & -.703 & NDI SRM $=0.58$ \\
\hline \multicolumn{7}{|l|}{ Nonsurgical Studies } \\
\hline \multirow[t]{5}{*}{ Moses et $\mathrm{al}^{15}$} & Neck pain & 130 & $\mathrm{PF}$ & NDI & -.771 & \\
\hline & & & & VAS Neck & -.428 & \\
\hline & & & & VAS Back & -.337 & \\
\hline & & & & VAS Arm & -.333 & \\
\hline & & & & VAS Leg & -.302 & \\
\hline
\end{tabular}

Abbreviations: ES, effect size; mJOA, modified Japanese Orthopaedic Association scale; NDI, Neck Disability Index; PB, pain behavior; PF, physical function; PIf, pain interference; PIn, pain intensity; Post-op, postoperative; Pre-op, preoperative; SRM, standard response mean; VAS, visual analog scale.

${ }^{a}$ Papuga et al reported a total of 283 patients, but did not specify the number of cervical patients vs. lumbar patients.

In addition, when comparing PROMIS measures with ODI measures at different follow-up time points, PROMIS PF and PIf showed greater correlation as follow-up time increased. PROMIS PF demonstrated increasing correlation with ODI, from baseline or preoperative scores $(|\mathrm{r}|=.53-.76)$ to 3-month postoperative scores $(|\mathrm{r}|=.74-.85)$ to 6month postoperative scores $(|\mathrm{r}|=.80-.84)$. PROMIS PIf demonstrated a similar pattern of increasing correlation with ODI with greater follow-up time, from baseline or preoperative scores $(|\mathrm{r}|=.59-.61)$ to 3-month postoperative scores $(|\mathrm{r}|=.79)$ to 6 month postoperative scores $(|\mathrm{r}|=.83)$.

\section{Global Health and Mental Health}

When comparing PROMIS with legacy measures of global health (Table 4), PROMIS PF was moderately to strongly correlated with the Short Form (SF)-12 $(|\mathrm{r}|=.50-.85)$ and strongly correlated with the SF-36 physical function domains (PFD; $|\mathrm{r}|$ $=.807)$, whereas the SF-12 moderately strongly correlated with PROMIS PIn $(|\mathrm{r}|=.34-.67)$ and PB $(|r|=.44-.47)$.
Three studies reported on PROMIS anxiety and depression scores compared with legacy mental health scores General Anxiety Disorder (GAD)-7, Patient Health Questionnaire (PHQ)-8, and modified Zung Depression Index (mZDI; Table 5). PROMIS depression scores strongly correlated with depression risk assessments PHQ-8 $(|\mathrm{r}|=.74-.79)$ and mZDI $(|\mathrm{r}|=.67)$, whereas PROMIS anxiety scores strongly correlated with the GAD-7 ( $|\mathrm{r}|=$ .71-.76).

\section{Time to Completion}

All studies that have reported on the time needed to complete PROM questionnaires in spine patients have shown that PROMIS requires significantly less time to complete than legacy measures (Table 6). ${ }^{8-10,17,18}$ On average, individual PROMIS domains required less time to complete (49.7-56 seconds) than ODI (176 seconds), NDI (190.3 seconds), SF-12 (214 seconds), and SF-36 PFD (99 seconds).

\section{Responsiveness}

The responsiveness of the PROMIS PF (effect size $[\mathrm{ES}]=0.35-1.42$, standard response mean 
PROMIS Spine Systematic Review

Table 3. Correlations of various PROMIS domains with lumbar spine and spinal deformity legacy measures.

\begin{tabular}{|c|c|c|c|c|c|c|}
\hline Study & Population & $\mathbf{n}$ & PROMIS & Legacy & Correlation & Responsiveness \\
\hline \multicolumn{7}{|l|}{ Surgical Studies } \\
\hline \multirow[t]{3}{*}{ Bhatt et al ${ }^{8}$} & Lumbar discectomy & 78 & $\mathrm{PF}$ & ODI & -.78 & $\mathrm{PF} \mathrm{ES}=1.42$ \\
\hline & & & PIn & ODI & .78 & PIn ES $=1.6$ \\
\hline & & & PB & ODI & .58 & $\mathrm{~PB} \mathrm{ES}=1.09$ \\
\hline \multirow[t]{9}{*}{ Haws et al ${ }^{11}$} & MIS TLIF & 74 & $\mathrm{PF}$ & ODI Pre-op & -.525 & \\
\hline & & & & ODI 3 mo & -.738 & \\
\hline & & & & ODI 6 mo & -.831 & \\
\hline & & & & VAS Back Pre-op & -.091 & \\
\hline & & & & VAS Back 3 mo & -.446 & \\
\hline & & & & VAS Back 6 mo & -.693 & \\
\hline & & & & VAS Leg Pre-op & -.333 & \\
\hline & & & & VAS Leg 3 mo & -.397 & \\
\hline & & & & VAS Leg 6 mo & -.452 & \\
\hline \multirow[t]{5}{*}{ Khechen et al ${ }^{14}$} & MIS Lumbar Microdiscectomy & 41 & $\mathrm{PF}$ & ODI Pre-op & .5735 & \\
\hline & & & & ODI $3 \mathrm{mo}$ & .8543 & \\
\hline & & & & VAS Back 3 mo & .6522 & \\
\hline & & & & VAS Leg Pre-op & .3964 & \\
\hline & & & & VAS Leg 3 mo & .6412 & \\
\hline \multirow[t]{6}{*}{ Papuga et $\mathrm{al}^{17}$} & Spine clinic & $283^{\mathrm{a}}$ & $\mathrm{PF}$ & ODI Pre-op & .7604 & \\
\hline & & & & ODI Post-op & .8468 & \\
\hline & & & PIf & ODI Pre-op & .6133 & \\
\hline & & & & ODI Post-op & .8907 & \\
\hline & & & $\mathrm{PB}$ & ODI Pre-op & .7226 & \\
\hline & & & & ODI Post-op & .8273 & \\
\hline \multirow[t]{6}{*}{ Patel et $\mathrm{al}^{18}$} & Lumbar stenosis & 98 & $\mathrm{PF}$ & ODI & -.58 & $\mathrm{PF} \mathrm{ES}=0.96$ \\
\hline & & & & ZCQ & -.61 & PIf $\mathrm{ES}=0.88$ \\
\hline & & & PIf & ODI & .73 & $\mathrm{~PB} \mathrm{ES}=0.70$ \\
\hline & & & & ZCQ & .66 & ODI $\mathrm{ES}=0.96$ \\
\hline & & & $\mathrm{PB}$ & ODI & .60 & $\mathrm{ZCQ} \mathrm{ES}=0.41$ \\
\hline & & & & ZCQ & .59 & \\
\hline \multirow[t]{6}{*}{ Purvis et $\mathrm{al}^{20}$} & Lumbar disc degeneration & 231 & $\mathrm{PF}$ & ODI & -.74 & \\
\hline & & & & BPI Pain If & -.51 & \\
\hline & & & & BPI BP & -.32 & \\
\hline & & & PIn & ODI & .73 & \\
\hline & & & & BPI Pain If & .61 & \\
\hline & & & & BPI BP & .33 & \\
\hline \multirow[t]{2}{*}{ Sharma et $\mathrm{al}^{22}$} & Lumbar surgery & 52 & $\mathrm{PF}$ & ODI Pre-op & -.753 & PF SRM - 0.84 \\
\hline & & & & ODI 3 mo & -.773 & ODI SRM - 0.70 \\
\hline \multicolumn{7}{|c|}{ Mixed or Nonsurgical Studies } \\
\hline \multirow[t]{3}{*}{ Bernstein et $\mathrm{al}^{7}$} & Adult spinal deformity & 163 & $\mathrm{PF}$ & SRS-30 & .78 & \\
\hline & & & PIf & SRS-30 & -.70 & \\
\hline & & & $\mathrm{D}$ & SRS-30 & -.80 & \\
\hline Brodke et al ${ }^{10}$ & Back/leg pain & 1607 & $\mathrm{PF}$ & ODI & -.81 & \\
\hline \multirow[t]{6}{*}{ Hung et $\mathrm{al}^{13}$} & Spinal disorders & 763 & $\mathrm{PF}$ & ODI Baseline & -.66 & PF SRM - 0.97-1.31 \\
\hline & & & & ODI 3 mo & -.76 & PIf SRM - 0.94-1.16 \\
\hline & & & & ODI $6 \mathrm{mo}$ & -.80 & ODI SRM - 1.16-1.33 \\
\hline & & & PIf & ODI Pre-op & .59 & PF ES - 0.98-1.11 \\
\hline & & & & ODI 3 mo & .79 & PIf ES - 1.29-1.39 \\
\hline & & & & ODI 6 mo & .83 & ODI ES - 1.03-1.08 \\
\hline \multirow{6}{*}{ Raad et $\mathrm{al}^{21}$} & Adult spinal deformity & 123 & $\mathrm{PF}$ & ODI & $.76^{\mathrm{b}}$ & PF ES --0.29 \\
\hline & & & & SRS-22r & $.51^{\mathrm{b}}$ & PIf ES - 0.80 \\
\hline & & & PIf & ODI & $.77^{\mathrm{b}}$ & Ax ES -0.46 \\
\hline & & & & SRS-22 & $.60^{\mathrm{b}}$ & ODI ES - 0.67 \\
\hline & & & $A x$ & ODI & $.52^{\mathrm{b}}$ & SRS ES --0.23 \\
\hline & & & & SRS-22r & $.73^{b}$ & \\
\hline
\end{tabular}

Abbreviations: Ax, Anxiety; BPI BP, Brief Pain Inventory Back Pain; BPI Pain If, Brief Pain Inventory Pain Interference; D, depression; MIS, minimally invasive surgical; TLIF, transforaminal lumbar interbody fusion; ODI, Oswestry disability index; PB, pain behavior; PF, physical function; PIf, pain interference; PIn, pain intensity; Postop, postoperative; Pre-op, preoperative; VAS, Visual analog scale; SRS-22r, Scoliosis research society questionnaire; ZCQ, Zurich claudication questionnaire; ES, effect size; SRM, standard response mean.

${ }^{\text {a}}$ Papuga et al reported a total of 283 patients but did not specify the number of cervical patients vs lumbar patients.

${ }^{\mathrm{b}}$ Reported as Spearman correlations; all other correlations reported as Pearson correlations.

$[\mathrm{SRM}]=0.31-1.31)$, PIf $(\mathrm{ES}=0.8-1.39, \mathrm{SRM}=$ $0.78-1.16)$, and $\mathrm{PB}(\mathrm{ES}=0.7-1.09)$ was comparable to that of legacy measures ODI $(\mathrm{ES}=0.96-1.03$, $\mathrm{SRM}=0.7-1.33)$, NDI $(\mathrm{ES}=074-0.76, \mathrm{SRM}=$ $0.58-1.18)$ and SF-12 $(\mathrm{ES}=0.675) .{ }^{8,13,18,19,21,22}$

\section{Floor and Ceiling Effects of PROMIS}

Multiple included studies reported on floor and ceiling effects of various PROMIS domains. Reported floor effects for PROMIS PF $(0.0 \%-$ $3.86 \%),{ }^{9,10,18,23}$ PIf $\left.(0.0 \%-0.44 \%)\right)^{7,15,18}$ and PB 
Table 4. Correlations of various PROMIS domains with global health legacy measures.

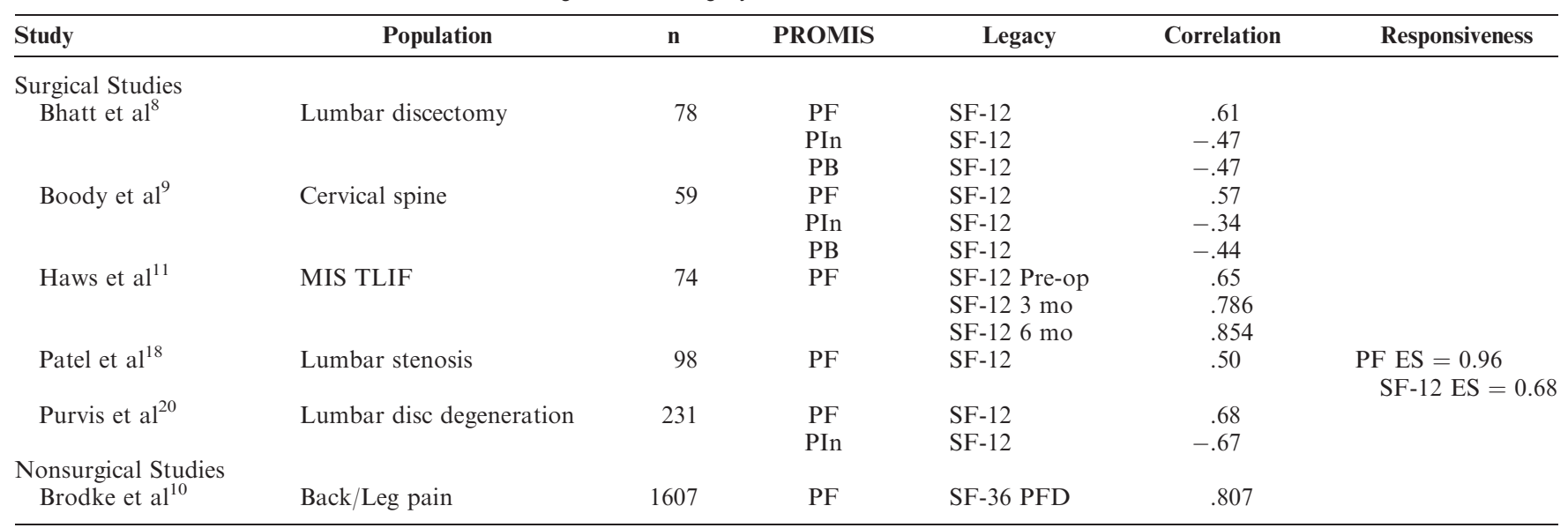

Abbreviations: ES, effect size; MIS, minimally invasive surgical; TLIF, transforaminal lumbar interbody fusion; PB, pain behavior; PF, physical function; PIn, pain intensity; SF-12, Short Form 12; SF-36 PFD, Short Form 36 physical function domains.

$(0.0 \%-1.0 \%)^{9,18}$ were comparable or less than those reported for SF-36 PFD $(23.65 \%),{ }^{10}$ ODI $\left(0 \%{ }^{-}\right.$ $44.24 \%),{ }^{9,10,18}$ NDI $(7.10 \%),{ }^{15}$ SF-12 $(0.0 \%),{ }^{9,18}$ and SRS-22r $(0.88 \%-1.32 \%){ }^{7} \quad$ Similarly, ceiling effects were relatively low for PROMIS PF $(0.0 \%-$ $1.7 \%), 9,10,18,23$ PIf $(0.0 \%-0.88 \%))^{7,15,18}$ and $\mathrm{PB}$ $(0.0 \%))^{9,18}$

\section{DISCUSSION}

As health care becomes more reliant on PROMs to evaluate treatments, numerous general and disease-specific measures have been put forth as means to assess the impact of various interventions across orthopedic pathologies. Nevertheless, these instruments are often cumbersome to complete and/ or lack the ability to compare across populations and pathologies. PROMIS is promoted as a fast, simple instrument with broad applicability, and it has become increasingly used in research and to assess clinical outcomes in orthopedics. ${ }^{2,4}$ The goal of this study was to systematically review the literature available comparing the psychometric properties of PROMIS and legacy measures in spinal populations.

Across all populations included in this review, we found PROMIS to have strong correlation with legacy outcome measures. In the cervical population, PROMIS PF, PIn, PIf, and PB all moderately to strongly correlated with the NDI $(|\mathrm{r}|=.47-.88)$ and PF strongly correlated with the mJOA $(|\mathrm{r}|=$ .61-.72). Among patients with lumbar pathology and adult spinal deformity, the PROMIS PF, PIn, PIf, and PB all strongly correlated with the ODI ( $|\mathrm{r}|$ $=.70-.76$ ), and PROMIS PF and PIf also strongly correlated with the SRS-22 and SRS-30 (average $|\mathrm{r}|$ $=.65$ ). Similarly, PROMIS measures correlated strongly with measures of global health (SF-12 and SF-36), as well as those assessing depression and anxiety, such as the PHQ-8, GAD-7, and mZDI.

It is important for the providers and reseachers in spine to be able to coordinate the use of PROMs in

Table 5. Correlations of PROMIS anxiety and depression scores with mental health legacy measures.

\begin{tabular}{|c|c|c|c|c|c|c|}
\hline Study & Population & n & PROMIS & Legacy & Correlation & Responsiveness \\
\hline \multicolumn{7}{|l|}{ Surgical Studies } \\
\hline \multirow[t]{4}{*}{ Purvis et $\mathrm{al}^{19}$} & \multirow[t]{4}{*}{ Anterior cervical spine } & \multirow[t]{4}{*}{148} & \multirow[t]{2}{*}{ Anxiety } & GAD-7 & .76 & \multirow{8}{*}{$\begin{array}{l}\text { Anxiety ES }=-0.55 \\
\text { Depression ES }=-0.24\end{array}$} \\
\hline & & & & PHQ-8 & .73 & \\
\hline & & & Depression & GAD-7 & .63 & \\
\hline & & & & PHQ-8 & .74 & \\
\hline \multirow{4}{*}{ Purvis et $\mathrm{al}^{20}$} & \multirow{4}{*}{ Lumbar disc degeneration } & \multirow{4}{*}{231} & Anxiety & GAD-7 & .71 & \\
\hline & & & & PHQ-8 & .73 & \\
\hline & & & Depression & GAD-7 & .64 & \\
\hline & & & & PHQ-8 & .79 & \\
\hline \multirow{3}{*}{$\begin{array}{l}\text { Nonsurgical Studies } \\
\text { Hung et } \mathrm{al}^{12}\end{array}$} & \multirow{3}{*}{ Spine clinic } & \multirow{3}{*}{316} & & & & \\
\hline & & & Anxiety & mZDI & .712 & \\
\hline & & & Depression & mZDI & .667 & \\
\hline
\end{tabular}

Abbreviations: GAD-7, General Anxiety Disorder-7; mZDI, modified Zung Depression Index; PHQ-8, Patient Health Questionnaire. 
PROMIS Spine Systematic Review

Table 6. Time in seconds needed to complete each patient-reported outcome measure.

\begin{tabular}{|c|c|c|c|c|c|c|c|c|c|}
\hline Study & Population & $\mathbf{n}$ & PF & PIn & PB & ODI & NDI & SF-36 PFD & $\overline{\text { SF-12 }}$ \\
\hline Bhatt et $\mathrm{al}^{8}$ & Lumbar discectomy & 78 & 48 & 36 & 54 & 162 & & & 180 \\
\hline Boody et $\mathrm{al}^{9}$ & Cervical spine & 59 & 66 & 72 & 54 & & 204 & & 246 \\
\hline Brodke et $\mathrm{al}^{10}$ & Spine & 1607 & 44 & & & 169 & & 99 & \\
\hline Owen et $\mathrm{al}^{16}$ & Cervical myelopathy & 60 & 56 & & & & & & \\
\hline Papuga et al ${ }^{17}$ & Spine & 283 & 36 & 48 & & 187 & 180 & & \\
\hline Patel et $\mathrm{al}^{18}$ & Lumbar stenosis & 98 & 48 & & 60 & 186 & 187 & & 216 \\
\hline Average time, s & & & 49.7 & 52 & 56 & 176 & 190.3 & 99 & 214 \\
\hline
\end{tabular}

Abbreviations: NDI, Neck Disability Index; ODI, Oswestry Disability Index; PB, pain behavior; PF, physical function; PIn, pain intensity; SF-12, Short Form 12; SF-36 PFD, Short Form 36 physical function domains.

outcome research so that results can be compared between different studies. Guzman et $\mathrm{al}^{5}$ reported in 2016 that the top 6 most frequently used PROMs in spine practices were the VAS, ODI, SF-36, mJOA, NDI, and SRS-22, highlighting that the use of these different disease-specific legacy measures resulted in inconsistencies and author-dependent modifications that could not be standardized..$^{5}$ Similarly, Winebrake et $\mathrm{al}^{24}$ evaluated outcome reporting in the setting of fusion for lumbar spinal stenosis, noting substantial variability across the literature and recommending efforts to standardize reporting of outcomes to facilitate comparison across surgical interventions and pathologies. The high variability in PROMs used in research led the National Institute of health to develop PROMIS. The findings in this study support the use of PROMIS in diverse spine populations and anatomic locations.

In addition to standardized use and broad applicability, another important factor in determining the effectiveness and utility of PROMs is the burden of the instrument to the patient. Because most of these PROMs forms are administered during clinic visits, ease of use and time to completion are critical components of their success. On average, the PROMIS PF required 49.7 seconds to complete, PROMIS PIn required 52 seconds, and PROMIS PB required 56 seconds. Comparatively, legacy measures ODI (176 seconds), NDI (190.3 seconds), SF-12 (214 seconds), and SF-36 PFD (99 seconds) required more time to complete. The findings in this study demonstrate the possible reduced burden of administration of the PROMIS domains, highlighting this key advantage over legacy measures. However, investigators may want to administer multiple questionnaires to assess different domains of health and should be aware of the additive time of multiple questionnaires.

With these findings in mind, we believe that PROMIS has several advantages over other instruments. Including PROMIS data consistently in spine outcomes will allow practitioners and researchers to easily review literature and compare outcomes across interventions and pathologies. ${ }^{25}$ As seen in this review, the PROMIS domains focused on physical function and pain were the most commonly studied because these address symptoms often primarily targeted in spine patients. However, if investigators wish to study other domains such as global or mental health, they may still be able to compare results across the different domains because the PROMIS scoring system is a T-score metric with a score of 50 being the mean for a reference population and a 10-point standard deviation in either direction. PROMIS can be administered using the short form or computer adaptive testing, allowing practitioners to use technology to easily distribute questionnaires, increase response rates, and further reduce the administrative burden in their clinics.

There are several limitations of our study. As a systematic review, our study is limited by the quality of the underlying studies that were analyzed. Several studies that were examined included heterogeneous populations undergoing widely varied treatments, limiting the applicability to any 1 pathology or intervention. Nevertheless, the goal of this study was to examine the use of PROMIS in the spine literature, comparing its use with legacy outcome measures generally, and where possible we attempted to provide study details and organize by pathology and type of intervention (eg, surgical vs nonsurgical). Furthermore, there are multiple studies included in this review that were published by the same group of authors, raising the possibility of observer bias among these studies. Last, given that PROMIS was developed relatively recently, there are a limited number of studies providing direct comparisons of PROMIS to legacy measures. Therefore, although this review covers a range of different spinal pathologies, numerous diagnoses are not included. Future studies reporting PROMIS 
are needed to provide a more comprehensive review of the spine literature.

\section{CONCLUSIONS}

PROMIS PF, PIn, PIf, and PB demonstrated strong correlations with disease-specific legacy measures NDI, mJOA, ODI, and SRS-22 and global health measures SF-12 and SF-36 in cervical, lumbar, and spinal deformity patients. The decreased time to completion and comparable responsiveness of PROMIS domains support more widespread use of PROMIS in spine outcome research.

\section{REFERENCES}

1. Fidai MS, Saltzman BM, Meta F, et al. Patient-Reported Outcomes Measurement Information System and legacy patient-reported outcome measures in the field of orthopaedics: a systematic review. Arthroscopy. 2018;34(2):605-614.

2. Porter ME. What is value in health care? $N$ Engl J Med. 2010;363(26):2477-2481.

3. McCormick JD, Werner BC, Shimer AL. Patientreported outcome measures in spine surgery. $J$ Am Acad Orthop Surg. 2013;21(2):99-107.

4. Brodke DJ, Saltzman CL, Brodke DS. PROMIS for orthopaedic outcomes measurement. J Am Acad Orthop Surg. 2016;24(11):744-749.

5. Guzman JZ, Cutler HS, Connolly J, et al. Patientreported outcome instruments in spine surgery. Spine. 2016;41(5):429-437.

6. Intro to PROMIS. National Institute of Health. 2020. Available at http://www.healthmeasures.net/exploremeasurement-systems/promis/intro-to-promis. Accessed February 10, 2020.

7. Bernstein DN, Papuga MO, Sanders JO, Rubery PT, Menga EN, Mesfin A. Evaluating the correlation and performance of PROMIS to SRS questionnaires in adult and pediatric spinal deformity patients. Spine Deform. 2019;7(1):118-124.

8. Bhatt S, Boody BS, Savage JW, Hsu WK, Rothrock NE, Patel AA. Validation of Patient-Reported Outcomes Measurement Information System computer adaptive tests in lumbar disk herniation surgery. $J$ Am Acad Orthop Surg. 2019;27(3):95-103.

9. Boody BS, Bhatt S, Mazmudar AS, Hsu WK, Rothrock NE, Patel AA. Validation of Patient-Reported Outcomes Measurement Information System (PROMIS) computerized adaptive tests in cervical spine surgery. $J$ Neurosurg Spine. 2018;28(3):268-279.

10. Brodke DS, Goz V, Voss MW, Lawrence BD, Spiker WR, Hung M. PROMIS PF CAT outperforms the ODI and SF-36 physical function domain in spine patients. Spine. 2017;42(12):921-929.

11. Haws BE, Khechen B, Guntin JA, Cardinal KL, Bohl DD, Singh K. Validity of PROMIS in minimally invasive transforaminal lumbar interbody fusion: a preliminary evaluation. J Neurosurg Spine. 2018;29(1):28-33.

12. Hung M, Stuart A, Cheng C, et al. Predicting the
DRAM mZDI using the PROMIS anxiety and depression. Spine. 2015;40(3):179-183.

13. Hung M, Saltzman CL, Voss MW, et al. Responsiveness of the Patient-Reported Outcomes Measurement Information System (PROMIS), Neck Disability Index (NDI) and Oswestry Disability Index (ODI) instruments in patients with spinal disorders. Spine J. 2019;19(1):34-40.

14. Khechen B, Haws BE, Patel DV, et al. PROMIS physical function score strongly correlates with legacy outcome measures in minimally invasive lumbar microdiscectomy. Spine. 2019;44(6);442-446.

15. Moses MJ, Tishelman JC, Stekas N, et al. Comparison of Patient Reported Outcome Measurement Information System (PROMIS) with Neck Disability Index (NDI) and visual analog scale (VAS) in patients with neck pain. Spine. 2019;44(3);E162-E167.

16. Owen RJ, Zebala LP, Peters C, McAnany S. PROMIS physical function correlation with NDI and mJOA in the surgical cervical myelopathy patient population. Spine. 2018;43(8):550-555.

17. Papuga MO, Mesfin A, Molinari R, Rubery PT. Correlation of PROMIS physical function and pain CAT instruments with Oswestry Disability Index and Neck Disability Index in spine patients. Spine. 2016;41(14):1153-1159.

18. Patel AA, Dodwad SM, Boody BS, et al. Validation of Patient Reported Outcomes Measurement Information System (PROMIS) computer adaptive tests (CATs) in the surgical treatment of lumbar spinal stenosis. Spine. 2018;43(21):15211528.

19. Purvis TE, Andreou E, Neuman BJ, Riley LH 3rd, Skolasky RL. Concurrent validity and responsiveness of PROMIS health domains among patients presenting for anterior cervical spine surgery. Spine. 2017;42(23):E1357E1365.

20. Purvis TE, Neuman BJ, Riley LH 3rd, Skolasky RL. Discriminant ability, concurrent validity, and responsiveness of PROMIS health domains among patients with lumbar degenerative disease undergoing decompression with or without arthrodesis. Spine. 2018;43(21):1512-1520.

21. Raad M, Jain A, Huang $M$, et al. Validity and responsiveness of PROMIS in adult spinal deformity: the need for a self-image domain. Spine J. 2019;19(1);50-55.

22. Sharma M, Ugiliweneza B, Beswick J, Boakye M. Concurrent validity and comparative responsiveness of PROMIS-SF versus legacy measures in the cervical and lumbar spine population: longitudinal analysis from baseline to postsurgery. World Neurosurg. 2018;115:E664-E675.

23. Hung M, Hon SD, Franklin JD, et al. Psychometric properties of the PROMIS physical function item bank in patients with spinal disorders. Spine. 2014;39(2):158-163.

24. Winebrake JP, Lovecchio F, Steinhaus M, Farmer J, Sama A. Wide variability in patient-reported outcomes measures after fusion for lumbar spinal stenosis: a systematic review. Global Spine J. 2020;10(2):209-4215.

25. Rihn JA, Currier BL, Phillips FM, Glassman SD, Albert TJ. Defining the value of spine care. J Am Acad Orthop Surg. 2013;21(7):419-426.

Disclosures and COI: Han Jo Kim has received royalties from Zimmer Blomet and K2MStryker and is a consultant for Alphatec. All other 
authors received no funding for this study and report no conflicts of interest.

Corresponding Author: Kelsey Young, Department of Orthopaedic Surgery, University of Pennsylvania, 3737 Market St, Philadelphia, PA 19104. Phone: (631) 338-6705; Fax: (215) 662-3340; Email: kelseyyyoung@gmail.com.

Published 0 Month 2021

This manuscript is generously published free of charge by ISASS, the International Society for the Advancement of Spine Surgery. Copyright (C) 2021 ISASS. To see more or order reprints or permissions, see http://ijssurgery.com.

\section{APPENDIX. SEARCH STRATEGIES FOR RESPECTIVE ACADEMIC DATABASES}

\section{PubMed Strategy}

("Promis"[tw] OR "Patient-Reported Outcomes Measurement Information System"[tw] OR "Patient-Reported Outcome Measurement Information System"[tw]) AND ("Spine" [Mesh] OR "Spinal Diseases"[mesh] OR "Back Pain"[mesh] OR "neck"[mesh] OR "Spine"[tw] OR "Spinal" [tw] OR "lumbar" $[\mathrm{tw}])$

\section{EMBASE Strategy}

(promis:ti,ab,de,tn,kw OR 'patient-reported outcomes measurement information system':ti,ab, de,tn,kw OR 'patient-reported outcome measurement information system':ti,ab, de, tn,kw OR 'patient reported outcomes measurement information system'/exp OR 'patient reported outcome measurement information system'/exp) AND ('spine'/exp
OR 'spinal disease'/exp OR 'backache'/exp OR 'neck'/exp OR spine:ti,ab, de,tn,kw OR spinal:ti,ab,de,tn,kw OR lumbar: ti,ab,de,tn,kw)

\section{Cochrane Library Strategy}

(Promis:ti,ab,kw OR "Patient-Reported Outcomes Measurement Information System": ti,ab,kw OR "Patient-Reported Outcome Measurement Information System":ti,ab,kw) AND ([mh Spine] OR [mh "Spinal Diseases"] OR [mh "Back Pain"] OR [mh neck] OR Spine: ti,ab,kw OR Spinal:ti,ab,kw OR lumbar: ti,ab,kw)

\section{Resources for Selecting PROMIS Forms}

A full list of PROMIS adult measures can be found here:

http://www.healthmeasures.net/exploremeasurement-systems/promis/intro-to-promis/ list-of-adult-measures

A manual for the PROMIS physical function instruments including characteristics of the short form, computer adaptive tests, and scoring guide can be found here:

http://www.healthmeasures.net/images/ PROMIS/manuals/PROMIS_Physical_ Function_Scoring_Manual.pdf

A manual for the PROMIS pain interference instruments including characteristics of the short form, computer adaptive tests, and scoring guide can be found here:

http://www.healthmeasures.net/images/ PROMIS/manuals/PROMIS_Pain_Interference_ Scoring_Manual.pdf 